\title{
Efeitos de um marcador temporal sobre respostas de escolha por maior atraso e magnitude do reforço em crianças
}

Tatiana Chagas Correia. Universidade Federal da Bahia.

Nilza Micheletto. Pontifícia Universidade Católica de São Paulo.

\section{Resumo}

O presente experimento verificou se a apresentação de um marcador temporal durante o período de atraso do reforço aumentaria as respostas no componente com maior magnitude e atraso do reforço (autocontrole), diminuindo as escolhas pelo componente de menor atraso e magnitude (impulsividade), em um esquema concorrente. Três valores de atraso do reforço foram investigados. Participaram nove crianças distribuídas em três condições manipuladas no período de atraso do reforço: relógio, não-relógio e blackout. Escolhas entre o componente de autocontrole e impulsividade ocorriam na tela de computador sendo consequencia dos pontos trocados por brindes. Encontrou-se autocontrole na primeira apresentação dos menores valores de atraso no componente de maior atraso, mas não com valores maiores, independentemente da condição em vigor. O marcador temporal não indicou ser uma condição que aumente as respostas de autocontrole. Tais respostas estiveram relacionadas aos valores do atraso.

Palavras-chave: autocontrole; impulsividade; noção de tempo.

\begin{abstract}
Effects of a time marker on responses of choice for further delay and magnitude of reinforcement in children. This experiment aimed to determine whether the presentation of a temporal marker during the delay of reinforcement would increase choice response related to the greater magnitude and delay of reinforcement component (self-control), reducing the choices of the shorter delay and small magnitude component (impulsivity). Three values of delay interval have been investigated. Nine children were assigned to three conditions manipulated in the delay period: clock, no clock and blackout. Choices between self-control and impulsiveness component occurred on the computer screen producing points exchanged for gifts. Self-control was found in the first presentation of the smaller lag values in longer delay component, but not to larger values, regardless of the condition in effect. The time counter has not indicated to be a condition that increases the self-control. Such responses were related to the delay values.
\end{abstract}

Keywords: self-control; impulsiviness; notion of time.

\section{Resumen}

Los efectos de un contador de tiempo sobre las respuestas de elección por más retraso y magnitud del reforzamiento en niños. El presente experimento evaluó si la presentación de un contador de tiempo en el período de retraso del reforzamiento aumenta las respuestas del componente con más magnitud y retraso del reforzamiento (auto-control), reducindo la elección del componente con menos retraso y magnitud (impulsividad), en una esquema competidor. Tres tipos de retraso del reforzamiento han sido investigados. Participaron nueve niños asignados en tres condiciones manejadas en el periodo de retraso de refuerzo: con reloj, sin reloj y blackout. Las elecciones entre el componente de autocontrol y impulsividad ocurrieron en la pantalla del ordenador consequenciadas por puntos cambiados después por regalos. Se ha encuentrado auto-control en la primera presentación de los tipos más pequeños de retraso en el componente con más magnitud y retraso del reforzamiento, pero el mismo no ha pasado con los tipos de retrasos más largos, independientemente de la condición en efecto. El contador de tiempo no ha indicado ser una condición de incremento de las respuestas de auto-control. Tales respuestas solo se han relacionado con los tipo de retraso.

Palabras clave: auto-control; impulsividad; noción de tiempo. 
O comportamento de autocontrole tem sido estudado a partir de diferentes perspectivas teóricas e experimentais, sendo que o presente estudo teve como base a perspectiva da Análise do Comportamento. Inúmeras pesquisas com tal base passaram a estudar comportamentos denominados de autocontrole por meio de esquemas concorrentes de reforçamento em que a escolha por um componente do esquema produz maior atraso e magnitude do reforço - resposta de autocontrole -, e a escolha por outro produz menor atraso e magnitude do reforço - resposta de impulsividade (Canavarros, 2009; Dixon \& Tibbetts, 2009; Gokey, Wilder, Welch, Collier, \& Mathisen, 2013; Menezes, 2007; Newquist, Dozier, \& Neidert, 2012; Passage, Tincani, \& Hantula, 2012; Reed \& Martens, 2011; Vollmer, Borrero, Lalli, \& Daniel, 1999). Pesquisadores têm buscado em tais estudos identificar variáveis que controlam tal fenômeno ou que possam favorecer o aumento de probabilidade de respostas de autocontrole.

Ressalta-se que os pesquisadores devem estar cientes de que estão estudando apenas parte do fenômeno ao investigarem o autocontrole através da manipulação da interação entre as variáveis atraso e magnitude do reforço. Tipos diferentes de autocontrole não são abarcados como, por exemplo, escolha por um estímulo menos aversivo de forma imediata no lugar de um estímulo mais aversivo no futuro. Entretanto, estudar autocontrole por meio da manipulação de atraso e magnitude torna possível a investigação sistemática de variáveis que controlam tal fenômeno, contribuindo para a sua compreensão. Recortes do fenômeno podem ser feitos, contanto que o pesquisador tenha clareza do âmbito de suas conclusões, principalmente ao tentar generalizar os dados obtidos a partir do experimento (Hanna \& Todorov, 2002).

Alguns estudos manipularam o aumento ou diminuição gradual dos valores de atraso do reforço (Fading do atraso do reforço), na contingência de maior atraso e magnitude, com o objetivo de aumentar a frequência de respostas de autocontrole (Binder, Dixon, \& Guezzi, 2000; Canavarros, 2009; Dixon \& Tibbetts, 2009; Gokey et al, 2013; Mazur \& Logue, 1978; Schweitzer \& Sulzer-Azaroff, 1988). Tais estudos indicaram que o fading do atraso do reforço aumenta a probabilidade de escolhas por reforçadores atrasados e de maior magnitude.

Atividades alternativas (jogos) que ocorrem durante o período de atraso do reforço também foram manipuladas em alguns estudos (Baquero, 2005; Gebrin,
2009; Menezes, 2007) com o objetivo de verificar se a introdução dessas atividades produziria um aumento das respostas de autocontrole. Encontrou-se no estudo de Menezes (2007) que os participantes apresentaram um padrão de respostas de autocontrole durante todo o experimento, independentemente do tipo de atividade durante o período de atraso do reforço. Entretanto, no estudo de Baquero (2005) e de Gebrim (2009), a maioria das crianças apresentou respostas de autocontrole apenas nas sessões iniciais do experimento.

Algumas pesquisas manipularam atividades relacionadas à passagem do tempo (marcador temporal) durante o período de atraso do reforço (Newquist et al., 2012; Sonuga-Barke, Lea, \& Webley, 1989, Vollmer et al., 1999). Sonuga-Barke et al. (1989) tiveram como objetivo do experimento investigar quais as variáveis que determinam a sensibilidade às mudanças no parâmetro atraso do reforço, tendo como hipótese o fato de que crianças menores não discriminam tais mudanças. Segundo tais autores, essas crianças se comportam em função da magnitude do reforço apenas, o que não lhes promove, necessariamente, uma maior quantidade total de reforço na sessão. Eles enfatizaram que, para que se produza uma maior quantidade de reforço na sessão, é essencial não somente a sensibilidade à magnitude do reforço, mas também a discriminação da passagem do tempo.

Os participantes foram distribuídos em dois grupos, havendo uma diferença na relação entre a modificação na tela do computador e a passagem do tempo, durante o período de atraso para cada grupo: alguns participantes foram expostos a mudanças gráficas, sistemáticas e ordenadas relacionadas à passagem do tempo (quadrado colorido na tela que aumentava com a passagem do tempo) - condição de relógio -, enquanto para os outros a mudança gráfica não era relacionada com a passagem do tempo (condição de não-relógio). Para os participantes da condição relógio, os quadrados mudavam a cada três segundos, sendo que a quantidade de espaço colorido na tela aumentava à medida que o tempo passava e o quadrado no centro da tela diminuía, enquanto que na condição não-relógio quadrados eram dispostos na tela, piscando de forma não relacionada à passagem do tempo.

Os resultados apontaram que a utilização do relógio pareceu ter pouco efeito na sensibilidade a aumentos do atraso nas crianças, ou seja, não houve diferenças no responder dos participantes entre as condições relógio e não-relógio. Sonuga-Barke et al. (1989) discutiram que 
uma hipótese para explicação de tal ocorrência é que o display disposto na condição relógio pode não ter sido um recurso efetivo para enfatizar o atraso do reforço. Entretanto, eles discutiram que alguns sujeitos expostos à condição relógio referiram-se às mudanças no display, relacionando-as com mudanças no período de espera do reforço maior, enquanto que sujeitos expostos à condição não-relógio não apresentaram tal relato.

Além disso, os autores também utilizaram cálculos estatísticos considerando a quantidade de fichas ganhas e identificaram que crianças expostas à condição relógio obtiveram uma quantidade maior de reforços totais. Isto revela que, embora os autores tenham considerado que o relógio tenha exercido pouco efeito em estabelecer que os participantes se comportassem em função dos parâmetros do atraso, a mudança na tela de forma sistemática referente à passagem do tempo teve algum efeito em relação à quantidade total de reforços obtidos. Este aspecto está especialmente relacionado com o presente estudo.

Vollmer et al. (1999) introduziram um cronômetro no período de atraso do reforço em conjunto com o procedimento de fading em um estudo com crianças com déficits de desenvolvimento e verificaram um aumento das respostas de autocontrole. Também foi encontrado um aumento de respostas de autocontrole no estudo de Grey, Healy, Leader e Hayes (2009) ao introduzirem um marcador temporal no período de atraso do reforço. Entretanto, num estudo realizado por Newquist et al. (2012) com criança com desenvolvimento típico, verificou-se que um marcador temporal (cronômetro) não produziu respostas de autocontrole.

Desta forma, os estudos de Vollmer et al. (1999) e de Grey et al. (2009), ambos realizados com crianças com desenvolvimento atípico, verificaram que o marcador temporal pode favorecer o aumento de respostas de autocontrole, Newquist et al. (2012) não identificaram tal efeito; e Sonuga-Barke et al. (1989) identificaram alteração do número de respostas reforçadas quando o marcador temporal estava em vigor. Tais divergências de resultados mostram que o efeito desse procedimento para favorecer a discriminação temporal no período de atraso não está claramente estabelecido.

Pesquisas que verifiquem se um marcador temporal pode aumentar as respostas de autocontrole podem contribuir na avaliação do possível controle exercido pela discriminação temporal no comportamento de escolha por reforçadores de maior atraso e magnitude em crianças.
Todo comportamento, na perspectiva da análise do comportamento, é determinado por contingências de sobrevivência, por contingências relacionadas à vida pessoal e às dispostas pelos grupos a que pertence. As contingências em que uma pessoa adquire comportamento na vida pessoal junto a seu grupo são relativamente acessíveis e podem ser manipuladas. (Skinner, p. 44). Neste trabalho, foram investigadas possíveis contingências relacionadas à vida pessoal que podem produzir autocontrole em crianças.

Segundo Skinner (1974), as diferenças de comportamento de uma criança ao longo dos anos não ocorrem só porque ela cresceu mas porque ela teve tempo de adquirir um repertório muito maior por meio da exposição a novas contingências de reforçamento e particularmente porque as contingências que afetam as crianças em diferentes idades são diferentes. O mundo infantil se "desenvolve" também. (p. 67) Pesquisas que investigam autocontrole por meio de atraso e magnitude têm identificado predominantemente respostas denominadas de impulsividade que foram revertidas por métodos que promovem alteração em tais escolhas para respostas de autocontrole (Pear, 2001). O conhecimento de variáveis que promovam respostas denominadas de autocontrole em crianças pode favorecer o desenvolvimento de práticas que auxiliem no dia a dia os indivíduos com frequentes respostas de impulsividade e de profissionais e familiares na interação com os mesmos. Várias pesquisas têm gerado alternativas de ação para profissionais e familiares para ajudar pessoas a evitar comportamentos que geram consequências nocivas atrasadas, como por exemplo, o comer excessivo de crianças ou adultos (Cruz, 2012; Ferster, Nurnberg, \& Levit, 1973), consumo de drogas, o consumo excessivo, o desperdício de recursos ambientais (Rachlin, 2000).

A proposta do presente estudo foi verificar se a apresentação de um marcador temporal durante o período de atraso do reforço aumentaria o autocontrole de crianças. Buscou-se avaliar se diferentes valores de atrasos estariam relacionados ao comportamento de autocontrole. Considerou-se como comportamento de autocontrole a escolha pelo componente de maior atraso e magnitude do reforço e como comportamento de impulsividade a escolha pelo componente de menor atraso e magnitude. A hipótese do estudo foi que o marcador temporal produziria mais escolhas por este componente devido ao desenvolvimento de um repertório comportamental de discriminação da passagem do tempo, assim como a hipótese de Sonuga-Barke et al. (1989). 
Efeitos de um marcador temporal sobre respostas de escolha por maior atraso e magnitude do reforço em crianças

\section{Método}

\section{Participantes}

Nove crianças com idade entre 8 e 10 anos, quatro do sexo masculino e cinco do feminino, foram selecionadas por conveniência para participarem do presente estudo. Cursavam entre a $1^{\circ}$ e $3^{\circ}$ ano do ensino fundamental em diferentes escolas particulares (sete crianças) e públicas (duas crianças) da cidade de Salvador (BA), todas com desenvolvimento típico. A coleta foi realizada no período de férias das crianças, selecionando-se as que apresentassem sensibilidade às alterações nos valores de atraso e magnitude do reforço (fase descrita no tópico procedimentos).

\section{Materiais e equipamentos}

Utilizaram-se os seguintes materiais: 1) uma mesa, na qual o notebook era colocado; 2) um notebook; 3) duas cadeiras (uma para o participante e outra para o experimentador); 4) brindes utilizados como reforçadores (apitos, bexigas, canetas coloridas, prendedores de cabelo, adesivos, carrinhos e aviõezinhos de plástico, bolas de gude, lápis de cor, giz de cera, massa de modelar, acessórios femininos de plástico) com valor financeiro aproximado.

O software desenvolvido para a presente pesquisa estava programado para apresentar no display do computador, três retângulos, sendo que os dois maiores (cada um medindo $3,6 / 4,8 \mathrm{~cm}$ ), na parte superior da tela, permaneciam lado a lado. Um deles tinha cor amarela e o outro azul. O terceiro retângulo era menor (1,8/2,9 $\mathrm{cm}$ ) e estava centralizado na parte inferior da tela, de cor vermelha. Estes três retângulos não eram apresentados simultaneamente. Além disso, havia dois contadores localizados dos dois lados do retângulo menor, os quais registraram o número de pontos recebidos como consequência de responder em cada um dos retângulos maiores. Os contadores tinham a cor do retângulo relacionado à resposta de escolha que estivesse do seu lado. O número de pontos era registrado com pequenos traços verdes ao lado de cada contador.

Na tela do computador, nos períodos de atraso do reforço, ocorria, a depender da condição experimental, uma de três configurações: 1) um círculo que sofria alterações relacionadas à passagem do tempo, de forma que o círculo fosse gradualmente preenchido por bolinhas coloridas piscantes; 2) um círculo com alterações não relacionadas à passagem do tempo em que bolinhas coloridas piscavam; 3) a tela permanecia completamente preta. Na Figura 1 está representado um esquema da tela do computador.

O software foi programado para registrar o número de tentativas em cada sessão, a latência da resposta de clicar, resposta de clicar, magnitude e o atraso do reforço, o número de pontos adquiridos por tentativa, as respostas em cada retângulo, bem como a hora em que a sessão ocorreu.

\section{Procedimentos}

Antes de serem iniciadas as sessões, foi solicitado aos responsáveis por cada participante assinarem o termo de consentimento livre e esclarecido. Além disso, solicitou-se o assentimento verbal das crianças antes do início das sessões.

No início de todas as sessões, os participantes eram conduzidos à sala experimental e eram feitos os seguintes comentários pela experimentadora "vamos brincar de um jogo bem legal!", "quanto mais fichas você ganhar, mais brinquedos poderá trocar". Utilizou-se uma instrução padronizada no início de cada sessão, na qual a experimentadora mostrava o que ocorreria na tela do computador à medida que explicava ao participante o funcionamento do software e o que o mesmo deveria fazer.

Estava em vigor um esquema concorrente-encadeado. Cada sessão experimental tinha 14 tentativas, sendo as quatro iniciais forçadas seguidas de 10 tentativas livres. Nas tentativas forçadas era apresentado na tela apenas um dos retângulos (azul ou amarelo), restringindo a resposta do participante a apenas este componente, com o objetivo de que o participante entrasse em contato com a consequência da escolha por cada componente. Os retângulos azul e amarelo eram apresentados alternadamente e os pontos adquiridos não eram contabilizados. Já nas tentativas livres (Figura 1), os dois componentes (azul e amarelo) eram apresentados, havendo assim a possibilidade de escolha entre os mesmos pelo participante.

As tentativas forçadas e livres eram compostas por três períodos. No período de escolha nas tentativas livres, apareciam na tela os retângulos azul e amarelo, com esquemas independentes (VI10s), com posição trocada randomicamente, estando em vigor um COD 3s se houvesse alternância entre os retângulos clicados; nas tentativas forçadas, a diferença era que aparecia apenas um dos componentes por tentativa e, consequentemente, não havia um COD. O retângulo amarelo sempre estava relacionado ao reforçador com menor atraso e 


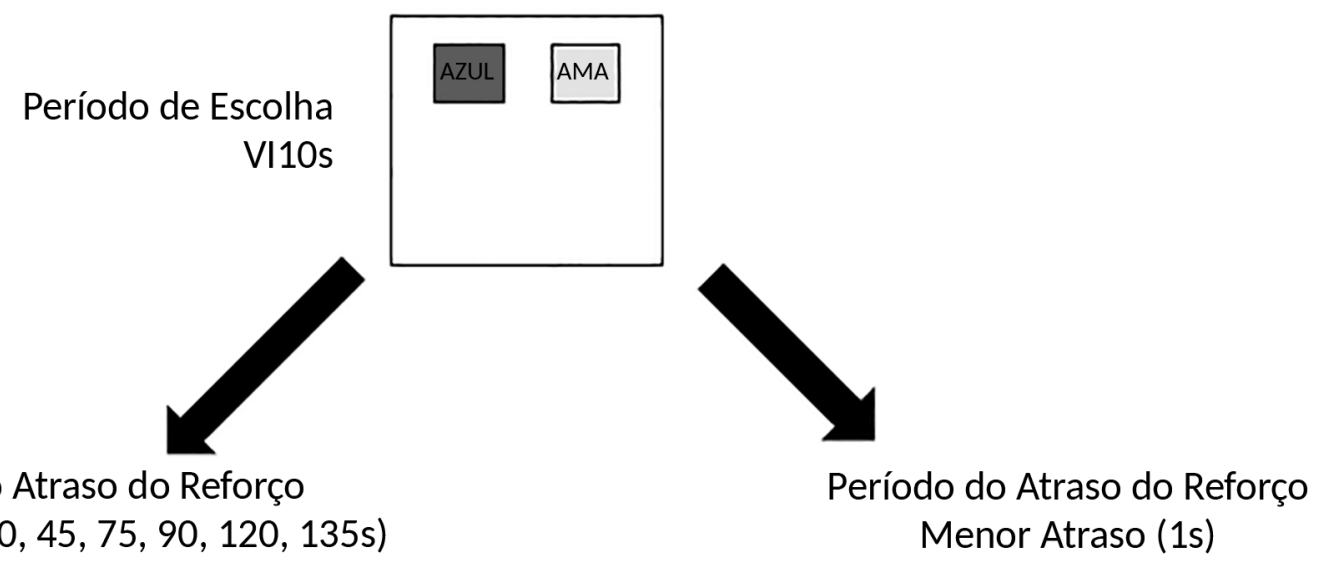

Maior Atraso (30, 45, 75, 90, 120, 135s)

Menor Atraso (1s)
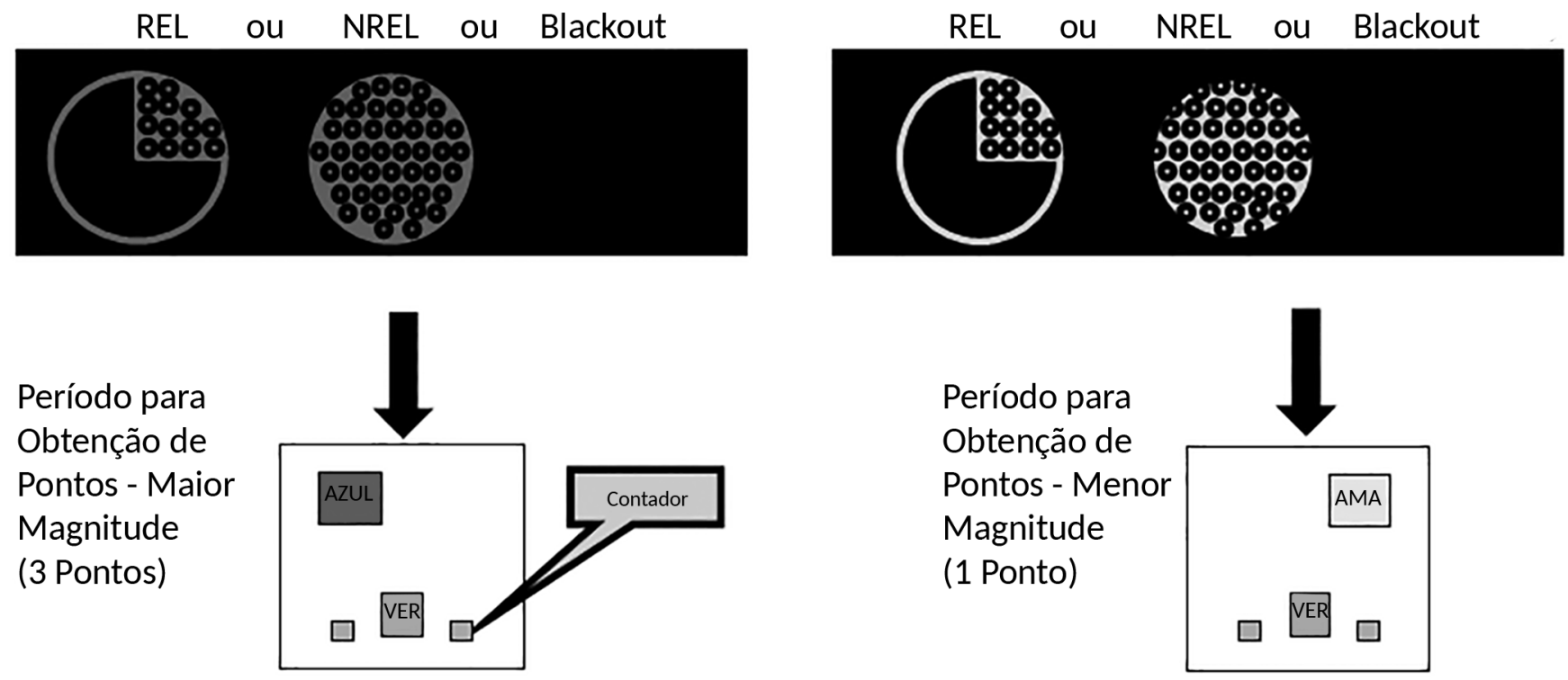

Figura 1. Configurações da tela do computador no período de escolha, no período de atraso do reforço e no período para obtenção de pontos, nas tentativas livres.

magnitude e o azul ao reforçador com maior atraso e magnitude. Após a passagem do período do esquema e uma resposta em um dos retângulos maiores, ocorria um som curto e iniciava-se o período de atraso do reforço.

No período de atraso do reforço, clicar com o mouse não produzia consequências e três possibilidades de configuração de tela poderiam ocorrer a depender da condição experimental: 1) um círculo da cor do retângulo escolhido que ia sendo preenchido no sentido horário, de forma sistemática em relação à passagem do tempo, com bolinhas que piscavam (Condição Relógio - REL); 2) um círculo da cor do retângulo escolhido, preenchido desde o início do período com bolinhas que piscavam, sem alterações relacionadas sistematicamente à passagem do tempo (Condição Não-Relógio - NREL) e; 3) tela preta (Condição Blackout).
O período de atraso do reforço era seguido pelo período disponível para obtenção de pontos. Neste, apareciam na tela o retângulo que havia sido escolhido, o contador de cada componente e o retângulo vermelho, que deveria ser clicado dentro de 10s para que os pontos fossem computados, os quais apareciam em forma de tracinhos verdes ao lado do contador, juntamente com um som de moedas caindo.

Utilizou-se um intervalo entre tentativas (ITI) de $3 \mathrm{~s}$, ou seja, ao final de cada tentativa havia um período de $3 s$ antes que iniciasse uma nova tentativa. Neste período aparecia a frase "Por favor, aguarde". No final da sessão apareciam os valores dos pontos que o participante havia adquirido naquela sessão. A quantidade de brindes recebida pelo participante era o valor de pontos na sessão dividido por três. Ele poderia escolher entre 
os diferentes tipos de brindes separados em seis caixas, variados a cada sessão. Perguntava-se, então, às crianças "Qual dos quadrados você mais gostou?" e "Por quê?", com objetivo de identificar se a criança apresentava um relato sobre o seu comportamento e o que o controlou coerente com o próprio comportamento na sessão.

Inicialmente, todos os participantes (T1, T2, T3, T4, T5, T6, T7, T8 e T9) foram expostos às fases de avaliação da sensibilidade a diferenças de atraso (Fase 1) e de magnitude (Fase 2) e, a seguir, às fases de manipulação simultânea de atraso e magnitude do reforço (Fases 3 e 4).

a) Fase de avaliação da sensibilidade à diferença de atraso do reforço foram utilizados diferentes valores de atraso em cada componente (30s para um e 1s para o outro) e mantida a magnitude do reforço baixa e constante (1 ponto) para os dois componentes. Caso o participante não atingisse o critério de estabilidade ( $80 \%$ de respostas de escolha no mesmo componente relacionado ao menor atraso em duas sessões consecutivas) em até seis sessões, os valores de atraso do reforço no componente de maior atraso e magnitude eram alterados de 30, 75 e 120s para 45, 90 e $135 \mathrm{~s}$, no restante do experimento.

b) Fase de avaliação da sensibilidade à diferença de magnitude do reforço foram utilizados diferentes valores de magnitude (1 ponto no componente amarelo e 3 no azul) e mantido o mesmo valor de atraso de reforço (1s) nos dois componentes. Utilizou-se o mesmo critério de estabilidade ( $80 \%$ das respostas consecutivas no componente relacionado à maior magnitude).

A seguir, todos os participantes foram expostos a duas fases em que manipulou-se simultaneamente atraso e magnitude do reforço.

c) Fases de manipulação simultânea de atraso e magnitude do reforço (Fases 3 e 4), nas quais o maior atraso estava ligado a maior magnitude do reforço e o menor atraso estava relacionado a menor magnitude do reforço. $O$ atraso do reforço relacionado ao componente de menor magnitude (retângulo amarelo; magnitude constante de 1 ponto) sempre era de 1s. Já o atraso do reforço relacionado ao componente de maior magnitude (retângulo azul; magnitude de 3 pontos) variou entre 30,75 e 120 s, ou 45,90 e $135 \mathrm{~s}$ caso o (a) participante não tivesse atingido o critério de estabilidade na fase de avaliação da sensibilidade à diferença de atraso com 30s de atraso no componente de maior magnitude. As sequências de apresentação dos atrasos foram: $1^{\text {a }) ~ 30, ~} 75$ e 120s, $2^{\mathrm{a})} 75,30,120$ s e $3^{\mathrm{a}}$ ) 120, 30, 75s. Assim, já que eram nove participantes, cada sequência de atraso foi apresentada a três deles, sendo que em cada condição estavam presentes as três sequências de atraso.

Os participantes T1, T2 e T3 foram expostos na Fase 3 à Condição Relógio - REL e na Fase 4 à Condição Não-relógio - NREL, com objetivo de comparar o desempenho dos mesmos participantes numa fase com marcador temporal e em outra sem o mesmo. Os participantes T4, T5 e T6 foram expostos na Fase 3 à Condição Não-relógio - NREL e na Fase 4 à Condição Relógio - REL, com objetivo de verificar se variações no desempenho dos mesmos estariam relacionadas a efeitos de ordem. Já os participantes T7, T8 e T9 foram expostos nas Fases 3 e 4 à Condição Blackout, para que todos os participantes fossem expostos ao mesmo número de condições. O objetivo desta condição foi verificar se uma tela sem alterações gráficas produziria desempenho diferente das Condições REL e NREL. Na Tabela 1 são apresentadas as fases, condições e sequência de valores de atraso do reforço às quais cada participante foi exposto ao longo do experimento.

\section{Resultados}

Todos os participantes mostraram sensibilidade a diferenças de atraso e magnitude do reforço nas condições de avaliação da sensibilidade a diferenças em tais variáveis. A Tabela 2 apresenta a preferência pelo componente de autocontrole ou impulsividade dos participantes nas duas fases em que houve variação simultânea de atraso e magnitude. As células acinzentadas denotam preferência (atingir o critério de estabilidade em determinado componente) do participante pelo componente de autocontrole e as não acinzentadas pelo de impulsividade.

Observa-se nas três condições uma preferência pelo componente de autocontrole somente quando os valores do atraso neste componente eram os menores ( 30 ou 45s) e na primeira vez em que tal valor de atraso era apresentado - com exceção de T2 e T9 que não apresentaram preferência pelo componente de maior atraso e magnitude com nenhum valor de atraso e T8 que apresentou tal preferência com o primeiro valor de 
Tabela 1. Apresentação dos Valores de Atraso e Magnitude (p)do Reforço nas Fases 1 e 2 e das Condições e Sequência de Valores de Atraso no Componente de Maior Atraso e Magnitude nas Fases 3 e 4, para cada Participante ao Longo do Experimento

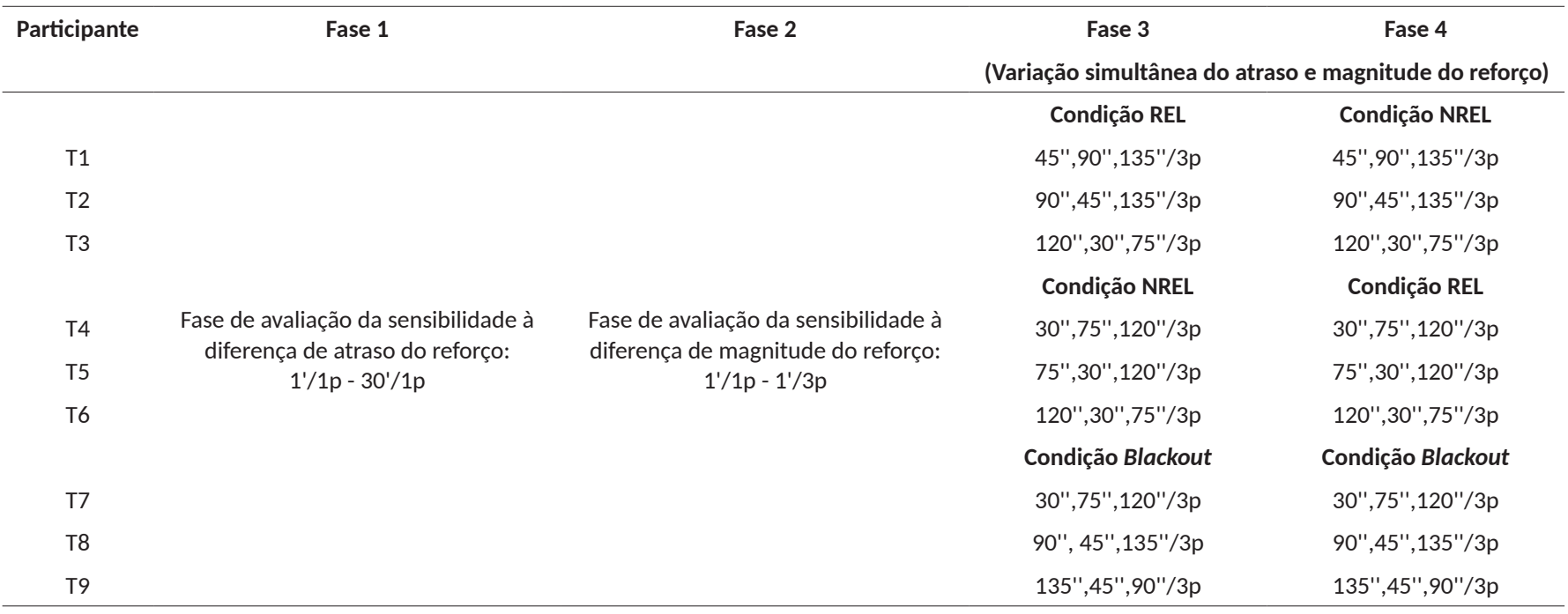

Tabela 2. Preferência pelo Componente de Autocontrole (Células Preenchidas com Cinza) ou Impulsividade (Células não Preenchidas) dos Participantes em Cada Fase de Variação Simultânea de Atraso e Magnitude

\begin{tabular}{|c|c|c|c|c|c|c|}
\hline \multirow{2}{*}{$\begin{array}{c}\text { Participante } \\
\text { T1 }\end{array}$} & \multicolumn{3}{|c|}{$1^{\text {a }}$ Fase de variação simultânea de atraso e magnitude (Fase 3) } & \multicolumn{3}{|c|}{$2^{a}$ Fase de variação simultânea de atraso e magnitude (Fase 4) } \\
\hline & REL 45s & REL90s & REL 135s & NREL 45s & NREL 90s & NREL 135s \\
\hline $\mathrm{T} 2$ & REL 90s & REL 45s & REL 135s & NREL 90s & NREL 45s & NREL 135s \\
\hline T4 & NREL 30s & NREL 75s & NREL 120s & REL 30s & REL 75s & REL 120s \\
\hline T5 & NREL 75s & NREL 30s & NREL 120s & REL 75s & REL 30s & REL 120s \\
\hline T8 & BLA 90s & BLA 45s & BLA 135s & BLA 90s & BLA 45s & BLA 135S \\
\hline T9 & BLA 135s & BLA 45s & BLA 90s & BLA 135s & BLA 45s & BLA 90s \\
\hline
\end{tabular}

atraso apresentado, que no caso foi 90s. Apenas T4 apresentou preferência pelo componente de maior atraso e magnitude do reforço nas duas vezes em que o valor do atraso neste componente era o menor (30s).

Sendo assim, o padrão de respostas de escolha do componente de autocontrole não se manteve nas condições com valores de atraso 75, 90, 120 e 135s e nem na reapresentação do menor valor (30 ou 45s) para a maioria dos participantes, independentemente da condição (REL, NREL ou Blackout) em vigor. Portanto, as variáveis relógio, não-relógio e o blackout produziram dados semelhantes.

Três participantes foram expostos ao aumento progressivo dos valores de atraso (45s, 90s, 135s para T1 e 30s, 75s, 120s para T4 e T7) nas fases experimentais em que foram manipulados simultaneamente atraso e magnitude do reforço, o que permitiu avaliar se tal aumento favorecia ou mantinha padrões de autocontrole. A Figura 2 apresenta a porcentagem de respostas de es- colha no componente de maior atraso e magnitude para estes participantes nos três valores de atrasos avaliados (menor, intermediário e maior). Na legenda da figura, encontram-se a sequência de condições a que cada participante foi exposto, juntamente com a sequência de valores de atraso. Comparando-se o desempenho desses participantes, verificou-se que o padrão de autocontrole identificado para a maioria dos participantes nos menores valores de atraso não se manteve com o aumento gradual do tempo de atraso do reforço.

Desta forma, o Fading do atraso do reforço (aumento gradual do tempo de atraso) não manteve um padrão de respostas de escolha no componente de autocontrole identificado no menor valor de atraso a que os participantes foram expostos.

Outro aspecto observado no desempenho dos participantes ao longo das sessões foi que os mesmos, caso se expusessem a um número de sessões igual ou maior do que quatro, poderiam obter mais pontos 


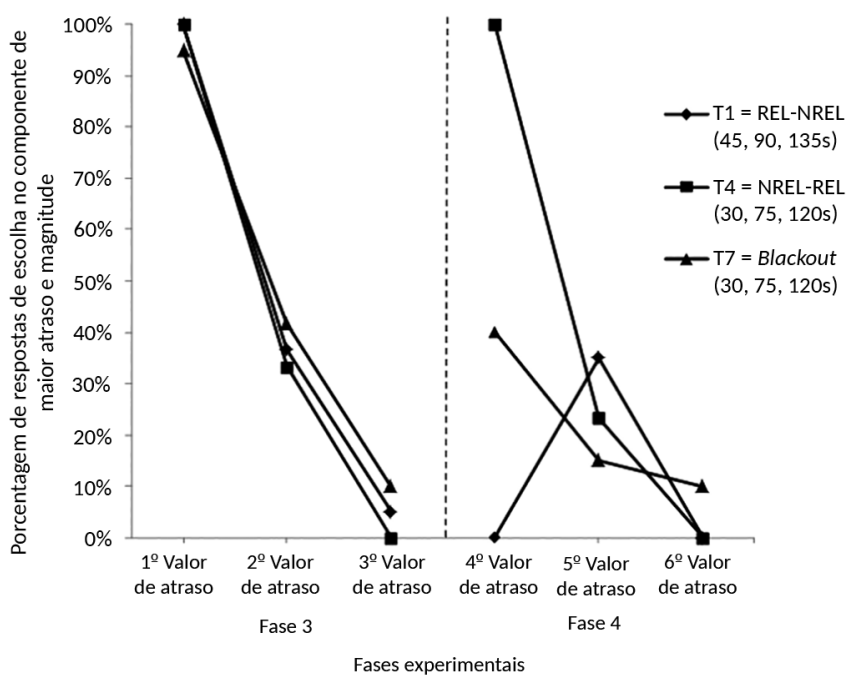

Figura 2. Porcentagem de respostas de escolha no componente de maior atraso e magnitude nas Fases 3 e 4, dos participantes expostas à sequência de aumento progressivo do atraso (T1, T4 e T7).

numa condição do que se atingissem o critério de estabilidade no componente de autocontrole. Na Tabela 3, foram apresentados o número de sessões ocorridas para atingir o critério de estabilidade e o número de pontos adquiridos em cada condição, de todos os participantes. Destacaram-se em cinza as condições em que os participantes atingiram o critério de estabilidade no componente de autocontrole.
Pode-se notar na Tabela 3 que os participantes que atingiram o critério de estabilidade no componente de autocontrole ganharam, em geral, entre 48 e 60 pontos, numa condição. Entretanto, a maior parte dos participantes que flutuaram entre respostas no componente de autocontrole e no de impulsividade ao longo da sessão, levando a partir de quatro sessões para atingir o critério de estabilidade, ganharam mais pontos naquela condição do que se tivessem apresentado um padrão de respostas de autocontrole na mesma. Pode-se observar, como exemplo, T1 na condição REL90s que realizou seis sessões, atingiu o critério de estabilidade no componente de impulsividade, mas adquiriu 100 pontos. T3 na condição REL120s que realizou cinco sessões atingiu o critério no componente de impulsividade, mas adquiriu 80 pontos no total da condição. T6 na condição REL30s que realizou cinco sessões atingiu o critério no componente de impulsividade, mas adquiriu 94 pontos. Outro participante que também apresentou esse padrão foi T7 na condição Blackout 30s a qual atingiu o critério no componente de impulsividade com 17 sessões, porém adquiriu 324 pontos. Esses resultados indicam que atingir o critério de estabilidade com um padrão de respostas de escolha pelo componente de maior atraso e magnitude do reforço não produzia uma maior quantidade de reforços nas condições, sendo o comportamento de alguns participantes sensível a isso.

Tabela 3. Número de Sessões e o Número de Pontos Adquiridos em Cada Condição de Todos os Participantes. Destacaram-se as Condições em que Cada Participante Atingiu o Critério de Estabilidade no Componente de Autocontrole

\begin{tabular}{|c|c|c|c|c|c|c|c|c|c|}
\hline Participante & Condição & № de sessões & Pontos & Condição & № de sessões & Pontos & Condição & № de sessões & Pontos \\
\hline $\mathrm{T} 1$ & REL 45s & 2 & 60 & REL 90s & 6 & 100 & REL 135s & 2 & 24 \\
\hline $\mathrm{T} 1$ & NREL 45s & 2 & 20 & NREL 90s & 4 & 68 & NREL 135s & 2 & 20 \\
\hline $\mathrm{T} 2$ & REL 90s & 2 & 20 & REL 45s & 2 & 20 & REL 135s & 2 & 20 \\
\hline $\mathrm{T} 2$ & NREL 90s & 2 & 22 & NREL 45s & 2 & 20 & NREL 135s & 2 & 20 \\
\hline T3 & REL 30s & 2 & 54 & REL 75s & 4 & 68 & REL 120s & 5 & 80 \\
\hline T3 & NREL30s & 4 & 78 & NREL 75s & 2 & 22 & NREL $120 \mathrm{~s}$ & 2 & 22 \\
\hline T4 & NREL 30s & 2 & 60 & NREL 75s & 3 & 44 & NREL 120s & 2 & 20 \\
\hline $\mathrm{T} 4$ & REL 30s & 2 & 60 & REL 75s & 3 & 50 & REL 120s & 2 & 19 \\
\hline T5 & NREL 30s & 5 & 124 & NREL 75s & 4 & 58 & NREL 120s & 3 & 42 \\
\hline T5 & REL 30s & 3 & 50 & REL 75s & 4 & 52 & REL 120s & 2 & 20 \\
\hline T6 & NREL 30s & 2 & 52 & NREL 75s & 2 & 24 & NREL 120s & 2 & 21 \\
\hline T6 & REL 30s & 5 & 94 & REL 75s & 2 & 26 & REL 120s & 2 & 20 \\
\hline T7 & Blackout 30s & 2 & 58 & Blackout 75s & 6 & 100 & Blackout 120s & 2 & 24 \\
\hline T7 & Blackout 30s & 17 & 324 & Blackout 75s & 4 & 51 & Blackout 120s & 2 & 24 \\
\hline $\mathrm{T} 8$ & Blackout 45s & 3 & 46 & Blackout 90s & 2 & 48 & Blackout 135s & 2 & 20 \\
\hline T8 & Blackout 45s & 2 & 20 & Blackout 90s & 2 & 24 & Blackout 135s & 2 & 22 \\
\hline T9 & Blackout 135s & 2 & 20 & Blackout 45s & 2 & 20 & Blackout 90s & 2 & 20 \\
\hline T9 & Blackout 135s & 2 & 20 & Blackout 45s & 2 & 22 & Blackout 90s & 2 & 20 \\
\hline
\end{tabular}




\section{Discussão}

O objetivo do presente estudo foi verificar se a introdução de um marcador temporal (relógio) nos períodos de atraso do reforço poderia favorecer o desenvolvimento de um padrão de escolha pelo componente de autocontrole. Pôde-se também avaliar se o aumento progressivo do tempo de atraso favorecia à manutenção ou estabelecimento de tal padrão. Comparando-se o desempenho dos participantes expostos ao relógio com o dos participantes expostos aos outros estímulos no período de atraso do reforço (não-relógio e blackout) pode-se concluir que o marcador temporal não favoreceu o desenvolvimento de um padrão de comportamento de autocontrole. Este padrão esteve especialmente relacionado ao tempo de atraso do reforço; tempos menores no componente de autocontrole produziram mais escolhas por tal componente na primeira vez que foram apresentados, em sete dos nove participantes. Para a maioria destes, este padrão não se manteve quando o mesmo valor de atraso no componente de autocontrole era apresentado pela segunda vez em uma nova condição.

Sonuga-Barke et al. (1989) e Newquist et al. (2012) verificaram que o marcador temporal utilizado no período de atraso do reforço não proporcionou um aumento nas respostas de autocontrole. Os dados do presente estudo corroboram com tais resultados, pois verificou-se que o marcador temporal não produziu mais respostas de autocontrole do que as outras condições manipuladas. Entretanto, tais resultados divergem dos encontrados por Grey et al. (2009), os quais verificaram que os participantes que foram expostos ao marcador temporal apresentaram mais respostas de autocontrole do que os que não foram. Esses resultados também divergem do estudo de Vollmer et al. (1999), o qual manipulou simultaneamente o fading e o marcador temporal e verificou o aumento de respostas de autocontrole. Todavia, deve-se considerar que tal resultado pode ter ocorrido devido à utilização do procedimento de Fading juntamente com o marcador temporal.

A semelhança de resultado nas três condições investigadas pode estar relacionada à sinalização do atraso presente em todas elas. Em um experimento, Richard (1981) comparou o desempenho de sujeitos (pombos) quando o atraso do reforço era sinalizado (apenas uma luz piloto) com quando o atraso era não-sinalizado, em diversos valores de atraso, sem manipular diferentes magnitudes. Ele encontrou um padrão de respostas similar entre os sujeitos expostos ao atraso sinalizado (diminuições moderadas no responder), sendo diferente em relação aos sujeitos expostos ao atraso não-sinalizado (diminuições acentuadas no responder), ou seja, o atraso sinalizado produziu efeito similar no responder dos sujeitos. Pode-se considerar que as condições Relógio, Não-Relógio e Blackout do presente experimento são correspondentes ao atraso sinalizado: 1) na condição Relógio, a tela do período de escolha era alterada para ser apresentado um relógio; 2) na condição Não-relógio, a tela do período de escolha era alterada para ser apresentado um círculo similar ao relógio não relacionado à passagem do tempo e; 3) na condição Blackout a tela era alterada para ser apresentada uma tela preta. Ou seja, nas três condições ocorriam sinalizações nos períodos de atraso do reforço, podendo assim ser considerados como atrasos sinalizados. Assim, o fato dessas três manipulações produzirem resultados similares é compatível com o resultado encontrado por Richard (1981). Novas investigações para verificar tal hipótese deveriam ser conduzidas em outros estudos.

Pesquisas em que se introduziram atividades durante o período de espera têm verificado que indivíduos expostos a diferentes atividades em tal período apresentaram padrões similares de comportamento (Baquero, 2005; Gebrin, 2009; Menezes, 2007; Sonuga-Barke et al., 1989), corroborando os resultados encontrados na presente pesquisa, os quais apontaram que o desempenho dos participantes foi similar, independentemente da condição em vigor. Pode-se supor que os estímulos apresentados na tela podem ter tido função semelhante a atividades propostas nestes estudos, já que havia uma alteração na tela para cada condição experimental.

Além disso, no estudo de Baquero (2005) e de Gebrin (2009), os participantes apresentaram um padrão de respostas de autocontrole apenas nas primeiras sessões experimentais, sendo que nas sessões seguintes, tais respostas ocorreram em baixa frequência. Os resultados do presente estudo corroboram tais resultados, já que os participantes apresentaram um padrão de escolhas de autocontrole apenas quando essas condições foram apresentadas na primeira sequência de valores do atraso e estavam relacionadas ao menor dos três valores investigados no componente de maior atraso e magnitude (30 ou 45s). Expor os participantes três sequencias de atraso estendeu muito o número de sessões, o que pode ter interferido neste resultado. Sugere-se que este aspecto seja controlado em estudos futuros.

Quando não há um período de tempo após o reforço no componente de menor atraso que compense o 
tempo entre este componente e o componente de maior atraso, à medida que se aumenta o atraso do reforço no componente de maior atraso, aumenta-se a frequência de respostas de escolha no componente com reforço menor e imediato (Sonuga-Barke et al., 1989). Desta forma, supõe-se que, no presente estudo, as altas frequências de respostas de escolha no componente de menor atraso e magnitude, quando o valor no componente de maior atraso era de $75 \mathrm{~s}, 90 \mathrm{~s}, 120$ s e $135 \mathrm{~s}$, estão relacionadas também com o fato de que não foi utilizado tal período de compensação entre os componentes - utilizou-se um intervalo de tempo fixo de $3 \mathrm{~s}$ entre as tentativas (ITI de 3s) para os dois componentes.

Outra hipótese que se deve levar em consideração para compreender a queda do responder na segunda apresentação da sequência de atraso é que a exposição ao jogo permitiu a discriminação de outras possibilidades de obtenção de pontos ao longo da fase que não a escolha do componente autocontrole (Rachlin, 2000).

Além disso, pode-se analisar a possível relação entre o número de sessões realizadas em cada condição, algumas vezes após flutuações entre os componentes, com o número de pontos adquiridos em cada condição. O experimento de Sonuga-Barke et al. (1989) objetivou avaliar se os participantes desenvolviam uma sensibilidade a variações simultâneas no atraso e magnitude do reforço. Utilizou-se um procedimento chamado titration, o qual tinha como objetivo testar a insensibilidade dos participantes às mudanças na densidade do reforço induzidas pelo atraso. Neste, para que os participantes obtivessem uma maior quantidade de reforçamento na sessão e não se submetessem a atrasos muito longos, eles não deveriam apresentar um padrão de respostas relacionado exclusivamente a um dos componentes. Eles deveriam, sim, apresentar variações do responder entre os dois componentes, o que indicaria uma sensibilidade às mudanças na densidade do reforço induzidas por mudanças nos valores de atraso.

No presente experimento, uma variação do responder entre os componentes (flutuações) também produzia maior quantidade de reforçamento total nas condições. Se o participante apresentasse o total de respostas de escolha num dos componentes, por duas sessões consecutivas, ele atingiria o critério de estabilidade, mudaria de fase e ganharia uma quantidade menor de pontos (do que se apresentasse flutuações) naquela fase.

Desta forma, há certa similaridade entre o presente experimento e o experimento realizado por Sonuga-Barke et al. (1989) no que se refere à aquisição de maior quanti- dade total de reforçamento produzida por um responder variado entre os componentes. Estes autores chamaram tal responder de adaptativo, pois evitava que o participante fosse exposto a longos atrasos e, ao mesmo tempo, produzia uma maior quantidade total de reforçamento.

Além de o marcador temporal não ter proporcionado o aumento de respostas de autocontrole, o aumento gradual do atraso do reforço no componente de autocontrole também não foi efetivo neste sentido, não corroborando os resultados encontrados por Mazur \& Logue (1978); Schweitzer \& Sulzer-Azaroff (1988); Canavarros (2009); Dixon \& Tibbetts (2009); Gokey et al. (2013). Tais estudos utilizaram aumentos progressivos de 5 em 5 ou de 10 em 10 segundos, enquanto que no presente estudo foram utilizados aumentos de $45 \mathrm{em} 45$ segundos. Desta forma, pode-se supor que este padrão de aumento dos valores do atraso pode ter contribuído para a não produção de escolhas no componente de maior atraso e magnitude quando esses valores foram aumentados.

Considerando-se estudos que manipularam diferentes variáveis, como Fading do atraso do reforço (Binder et al., 2000; Canavarros, 2009; Dixon \& Tibbetts, 2009; Gokey et al, 2013; Mazur \& Logue, 1978; Passage, Tincani, \& Hantula, 2012; Schweitzer \& Sulzer-Azaroff, 1988); reforços qualitativamente diferentes (Passage et al., 2012), atividades alternativas (Baquero, 2005; Gebrin, 2009; Menezes, 2007), marcador temporal no período de atraso (Grey et a., 2009; Vollmer et al., 1999), instruções sobre como responder em condições de espera (Luczynski \& Hanley, 2013), verifica-se que, dependendo do procedimento utilizado, crianças com diferentes idades e com desenvolvimento típico e atípico passam a apresentar respostas de autocontrole. Estes dados estão de acordo com a perspectiva skinneriana de que as variáveis que controlam o comportamento estão fora do organismo, na sua história ambiental e em seu ambiente mais imediato, as quais devem ser conhecidas o máximo possível para que se possa controla-las e prever os comportamentos (Skinner, 2007/1979).

\section{Conclusões}

Os resultados do presente estudo indicam que a introdução de um marcador temporal no período de espera não produziu resultados diferentes dos outros estímulos introduzidos em tal período. Por outro lado, quando os valores do atraso do reforço no componente de autocontrole eram os menores valores utilizados, a maioria dos participantes apresentou um padrão de 
respostas de autocontrole nas três condições manipuladas. Isso indica a possibilidade de um diálogo entre duas áreas de investigação: o estudo do autocontrole e o estudo sobre atraso sinalizado. Assim, sugerem-se que sejam realizadas pesquisas aplicadas que investiguem tal variável com objetivo de auxiliar pais e educadores no estabelecimento de autocontrole. Além disso, devem utilizar outros procedimentos que têm apresentado eficácia em outros estudos, como Fading do atraso do reforço e utilização de atividades alternativas durante o atraso. Como a sequência de aumentos progressivos do atraso do reforço não ocorreu gradualmente (aumento com valores menores), não se pôde verificar se um aumento gradual dos valores de atraso produziria autocontrole neste estudo. Sugere-se, assim, que pesquisas posteriores utilizem valores menores dos aumentos do atraso. Além disso, sugere-se que o critério de estabilidade utilizado envolva menor exigência para a mudança de fase do participante, já que alguns deles acabaram sendo expostos demasiadamente a períodos de atraso do reforço, produzindo preferência pelo componente de impulsividade no decorrer das sessões.

\section{Referências}

Baquero, R. G. (2005). Escolha no paradigma de autocontrole: efeito de reforçamento ou extinção na tarefa programada para o atraso do reforço (Dissertação de Mestrado não publicada). Universidade de Brasília, Brasília.

Binder, L. M., Dixon, M., \& Ghezzi, P. M. (2000). A procedure to teach self-control to children with attention deficit hyperactivity disorder. Journal of Applied Behavior Analysis, 33, 233-237. doi: 10.1901/ jaba.2000.33-233

Canavarros, D. A. P. (2009). Autocontrole: Um estudo sobre o efeito da manipulação do atraso do reforço a partir do procedimento de fading (Dissertação de Mestrado). Pontifícia Universidade Católica de São Paulo, São Paulo. Recuperado de http://www.sapientia.pucsp.br/ tde_busca/arquivo.php?codArquivo=9054

Cruz, M. T. M. (2012). Obesidade infantil: Uma proposta de tratamento comportamental (Dissertação de Mestrado). Pontifícia Universidade Católica de São Paulo, São Paulo. Recuperado de http://www.sapientia. pucsp.br/tde_busca/arquivo.php?codArquivo=14279

Dixon, M. R., \& Tibbetts, P. A. (2009). The effects of choice on self-control. Journal of Applied Behavior Analysis, 42, 243-252. doi: 10.1901/ jaba.2009.42-243

Ferster, C. B.; Nurnberg, J. I., \& Levit, E. E. (1973). The control of eating. In M. R. Golfried, \& M. Merbaum (Orgs.), Behavior change trough self control (pp. 195-212). Nova lorque, NY: Holt, Rinehart and Wiston.

Gebrin, A. (2009). Autocontrole e custo da resposta da tarefa programada no atraso de reforçamento (Dissertação de Mestrado). Universidade de Brasília, Brasília. Recuperado de http://bdtd.bce.unb.br/tedesimplificado/tde_busca/arquivo.php?codArquivo $=4835$
Gokey, K. M., Wilder, D. A., Welch, T., Collier, A., \& Mathisen (2013). Fading a concurrent activity during self-control training for children with autism. Journal of Applied Behavior Analysis, 46, 827-831. doi: 10.1002/jaba.77

Grey, I., Healy, O., Leader, G., \& Hayes, D. (2009). Using a Time Timer to increase appropriate waiting behavior in a child with developmental disabilities. Research in Developmental Disabilities, 30, 359-366. doi: 10.1016/j.ridd.2008.07.001

Hanna, E.S., \& Todorov, R. R. (2002). Autocontrole: um caso especial de comportamento de escolha. In J. Abreu-Rodrigues \& M. R. Ribeiro (Orgs.), Análise do comportamento: pesquisa teoria e aplicação (pp.175-184). Porto Alegre: Artmed.

Luczynski, K., \& Hanley, G. P. (2013). Prevention of problem behavior by teaching functional communication and self-control skills to prescholers. Journal of Applied Behavior Analysis, 46, 355-368. doi: 10.1002/jaba.44

Mazur, J. E., \& Logue, A. W. (1978). Choice in a "self-control" paradigm: Effects of a fading procedure. Journal of the Experimental Analysis of Behavior, 30, 11-17. doi: 10.1901/jeab.1978.30-11

Menezes, M. S. T. B. (2007). Autocontrole: Um estudo sobre o efeito da variação simultânea da magnitude e do atraso do reforço e da possibilidade de realização de atividades distrativas (Dissertação de Mestrado). Pontifícia Universidade Católica de São Paulo, São Paulo. Recuperado de http://www.sapientia.pucsp.br/tde_arquivos/3/ TDE-2007-07-10T09:02:18Z-3779/Publico/Mariana.pdf

Newquist, M. H., Dozier, C. L., \& Neidert, P. L. (2012). A comparison of the effects of brief rules, a timer, and preferred toys on self-control. Journal Applied Behavior Analysis, 45, 497-509. doi: 10.1901/ jaba.2012.45-497

Passage, M., Tincani, M., \& Hantula, D. A. (2012). Teaching self-control with qualitatively different reinforcers. Journal of Applied Behavior Analysis, 45, 853-857. doi: 10.1901/jaba.2012.45-853

Pear, J. J. (2001). The Science of learning. Philadelphia: Psychology.

Rachlin, H. (2000). The science of self-control. Cambridge: Harvard University

Reed, D. D., \& Martens, B. K. (2011). Temporal discouting predicts student responsiveness to exchange delas in a classroom roken system. Journal of Applied Behavior Analysis, 44, 1-18. doi: 10.1901/ jaba.2011.44-1

Richard, R. W. (1981). A comparison of signaled and unsignaled delay of reinforcement. Journal of Experimental Analysis of Behavior, 35, 145-152. doi: 10.1901/jeab.1981.35-145

Schweitzer, J. B., \& Sulzer-Azaroff, B. (1988). Self-control: Teaching tolerance for delay in impulsive children. Journal of Experimental Analysis of Behavior, 50, 173-186. doi: 10.1901/jeab.1988.50-173

Skinner, B. F. (1974). About Behaviorism. Nova lorque: Alfred A. Knopf.

Skinner, B. F. (2007). Ciência e comportamento humano. São Paulo: Martins Fontes. (Obra original publicada em 1979).

Sonuga-Barke, E. J. S., Lea, S. E. G., \& Webley, P. (1989). Children's choice: Sensitivity to changes in reinforcer density. Journal of the Experimental Analysis of Behavior, 51, 185-197. doi: 10.1901/ jeab.1989.51-185

Vollmer, T. R., Borrero, J. C., Lalli, J. S., \& Daniel, D. (1999). Evaluating self-control and impulsivity in children with severe behavior disorders. Journal Applied Behavior Analysis, 32 (4), 451-466. doi: 10.1901/ jaba.1999.32-451 
Efeitos de um marcador temporal sobre respostas de escolha por maior atraso e magnitude do reforço em crianças

Tatiana Chagas Correia, Mestra formada pelo Programa de Psicologia Experimental: Análise do Comportamento, da Pontifícia Universidade Católica de São Paulo (PUC-SP), Doutoranda do Programa de Pós-graduação em Psicologia da Universidade Federal da Bahia (UFBA) - ênfase em Psicologia do Desenvolvimento. Endereço para correspondência: Quintino de Carvalho, 203, Edf Pontevedra, Ap701, Jardim Apipema. Salvador - BA. Tel: (71) 9966-7096;

(71) 3018-7096. E-mail: tatianacorreia2013@gmail.com

Nilza Micheletto, Doutora formada pelo Programa de Psicologia Social da PUC-SP - Pontifícia Universidade Católica de São Paulo - PUC-SP, é Professora Titular da Pontifícia Universidade Católica de São Paulo - PUC-SP e coordenadora do Programa de Psicologia Experimental: Análise do Comportamento da PUC-SP. E-mail: nimicheletto@uol.com.br

Recebido em 17.Dez.14

Revisado em 09.Mar.15 Aceito em 22.Mai.15 